\title{
QCD thermodynamics with effective models
}

\author{
Bernd-Jochen Schaefer* \\ Institut für Physik, Karl-Franzens-Universität, A-8010 Graz, Austria \\ E-mail: bernd-jochen.schaefer@uni-graz.at
}

\section{Mathias Wagner}

Institut für Kernphysik, TU Darmstadt, D-64289 Darmstadt, Germany and ExtreMe Matter Institute EMMI, GSI Helmholtzzentrum für Schwerionenforschung GmbH, D-64291 Darmstadt, Germany

E-mail: mathias.wagner@physik.tu-darmstadt.de

\section{Jochen Wambach}

Institut für Kernphysik, TU Darmstadt, D-64289 Darmstadt, Germany and Gesellschaft für Schwerionenforschung GSI, D-64291 Darmstadt, Germany

E-mail: jochen.wambach@physik.tu-darmstadt.de

\begin{abstract}
In this talk we extend the Polyakov-quark-meson model to $N_{f}=2+1$ quark flavors and study its bulk thermodynamics at finite temperatures in mean-field approximation. Three different Polyakov-loop potentials are considered. Our findings are confronted to recent QCD lattice simulations of the RBC-Bielefeld and HotQCD collaborations. Furthermore, the finite chemical potential expansion of the quark-number susceptibility in a Taylor series around vanishing chemical potential is analyzed. By means of a novel algorithmic differentiation technique, we have calculated Taylor coefficients up to $24^{\text {th }}$ order in the model for the first time. This allows the systematic study of convergence properties of the Taylor series.
\end{abstract}

5th International Workshop on Critical Point and Onset of Deconfinement - CPOD 2009,

June 08 - 122009

Brookhaven National Laboratory, Long Island, New York, USA

${ }^{*}$ Speaker. 


\section{Introduction}

The deeper understanding of strongly interacting matter under extreme conditions plays a crucial role in many research programs. In particular, the search for possible (tri)critical endpoints in the phase diagram of strongly interacting matter is a major focus of, e.g., the planned CBM experiment at the FAIR facility. The underlying theory, QCD predicts several phases which are linked to certain phase transitions. For example, the confined, hadronic phase at low temperatures and chemical potentials (net quark densities) is separated from the deconfined quark-gluon plasma phase at high temperature and densities. Chiral and deconfinement aspects of these QCD phase transitions are the major focus of this talk.

For small temperatures and finite chemical potentials the chiral phase transition is probably of first-order while a crossover is expected at high temperatures and small chemical potentials. This suggests the existence of at least one critical endpoint (CEP) where the first-order transition line in the phase diagram terminates. At the CEP the transition is of second-order. The location and even the possible existence of additional endpoints in the phase diagram is still an open question (see e.g.[1]). Furthermore, the coincidence of the chiral and the deconfinement transition at vanishing chemical potentials and a possible confined but chirally symmetric phase, the quarkyonic phase, [2] at finite chemical potentials are much under debate.

Different regimes of the QCD phase diagram can be explored by employing various theoretical methods. Lattice QCD simulations are applicable at zero or imaginary chemical potentials but at finite real chemical potentials the fermion sign problem is still a considerable obstacle. At finite temperatures recent lattice simulations describe the QCD thermodynamics very trustfully since larger volumes and quark masses closer to their physical values are used. Despite this progress some lattice groups differ in their predictions even at vanishing chemical potentials. The RBCBielefeld group sees a coincidence of the chiral and deconfinement transition while the Wuppertal group obtains a larger critical temperature for the deconfinement transition $[3,4,5]$. Several extrapolations techniques towards small finite chemical potentials such as the reweighting method, imaginary chemical potential or a Taylor expansion around vanishing chemical potentials have been proposed (see [6] for an overview).

Another theoretical method is based on NJL-type or quark-meson models which incorporate chiral symmetry breaking in an effective manner. These models do not suffer from any limitations at finite chemical potentials or finite volumes but cannot address the deconfinement transition due to the lack of confinement. However, such types of models can be augmented with the Polyakov loop resulting in Polyakov-loop NJL-type (PNJL) or Polyakov-loop quark-meson (PQM) models. With these extended models both phase transitions become accessible.

In this talk we report on recent results from a chiral PQM model with $N_{f}=2+1$ quark flavors. We compare the bulk thermodynamics of this model for various Polyakov-loop potentials with newer QCD lattice data where the larger quark masses on the lattice are also explicitly considered in the model comparison.

Based on a novel numerical method, the algorithmic differentiation technique, higher derivatives of the thermodynamic potential can be extremely precise calculated [7]. This method, applied to the PQM model, allows us to investigate convergence properties of the Taylor expansion method used on the lattice. 


\section{Polyakov-quark-meson model for three quark flavors}

The Polyakov-quark-meson model for three quark flavors is a combination of the chiral linear $\sigma$-model with the Polyakov loop $\Phi(\vec{x})$, the thermal expectation value of a color traced Wilson loop in temporal direction [8]. Since the Polyakov loop in these types of effective models is used as a classical variable, implementation details of this quantity are not important. It serves as an order parameter of the center symmetry in the limit of infinitely heavy quarks. It is finite at high temperatures corresponding to the deconfined plasma phase, where the center symmetry is spontaneously broken and it vanishes in the confined, center-symmetric phase. However, in a system with dynamical quarks the center symmetry is always broken explicitly but $\Phi$ still seems to be a useful indicator of the confinement/deconfinement transition. The PQM Lagrangian consists of a quark-meson part and a Polyakov-loop potential $\mathscr{U}(\Phi, \bar{\Phi})$, which depends on the Polyakov-loop variable $\Phi$ and its hermitian conjugate $\bar{\Phi}$. The coupling of the background gauge field to the quarks is achieved by replacing the standard derivative $\partial_{\mu}$ in the quark-meson contribution with a covariant derivative $D_{\mu}=\partial_{\mu}-i A_{\mu}$ with $A_{\mu}=\delta_{\mu 0} A^{0}$. This leads to the Lagrangian

$$
\mathscr{L}_{\mathrm{PQM}}=\bar{q}\left(i \not D-g \phi_{5}\right) q+\mathscr{L}_{m}-\mathscr{U}(\Phi, \bar{\Phi}),
$$

where the interaction between the scalar $\left(\sigma_{a}\right)$ and the pseudoscalar $\left(\pi_{a}\right)$ meson nonets and the three quark flavors $q$ is implemented by an Yukawa-type vertex

$$
\phi_{5}=\frac{\lambda_{a}}{2}\left(\sigma_{a}+i \gamma_{5} \pi_{a}\right)
$$

The remaining, purely mesonic contribution reads

$$
\begin{aligned}
\mathscr{L}_{m}= & \operatorname{Tr}\left(\partial_{\mu} \phi^{\dagger} \partial^{\mu} \phi\right)-m^{2} \operatorname{Tr}\left(\phi^{\dagger} \phi\right)-\lambda_{1}\left[\operatorname{Tr}\left(\phi^{\dagger} \phi\right)\right]^{2}-\lambda_{2} \operatorname{Tr}\left(\phi^{\dagger} \phi\right)^{2} \\
& +c\left(\operatorname{det}(\phi)+\operatorname{det}\left(\phi^{\dagger}\right)\right)+\operatorname{Tr}\left[H\left(\phi+\phi^{\dagger}\right)\right],
\end{aligned}
$$

with the short-hand notation $\phi=\lambda_{a}\left(\sigma_{a}+i \pi_{a}\right) / 2$. The Polyakov loop potential $\mathscr{U}$ preserves the center symmetry of the pure Yang-Mills (YM) theory and several explicit choices are possible which we compare with each other in the following.

\subsection{Different choices of the Polyakov loop potential}

Several parameterizations of the Polaykov loop potential have been proposed in the last years, see [9]. The simplest choice is based on a Ginzburg-Landau ansatz and results in an expansion in terms of the order parameter

$$
\frac{\mathscr{U}_{\text {poly }}}{T^{4}}=-\frac{b_{2}}{4}\left(|\Phi|^{2}+|\bar{\Phi}|^{2}\right)-\frac{b_{3}}{6}\left(\Phi^{3}+\bar{\Phi}^{3}\right)+\frac{b_{4}}{16}\left(|\Phi|^{2}+|\bar{\Phi}|^{2}\right)^{2}
$$

with a temperature-dependent coefficient $b_{2}(T)=a_{0}+a_{1}\left(\frac{T_{0}}{T}\right)+a_{2}\left(\frac{T_{0}}{T}\right)^{2}+a_{3}\left(\frac{T_{0}}{T}\right)^{3}$. The parameters are adjusted to the pure gauge lattice data. An improved ansatz, motivated by the $S U(3)$ Haar measure, results in

$$
\frac{\mathscr{U}_{\mathrm{log}}}{T^{4}}=-\frac{1}{2} a(T) \bar{\Phi} \Phi+b(T) \ln \left[1-6 \bar{\Phi} \Phi+4\left(\Phi^{3}+\bar{\Phi}^{3}\right)-3(\bar{\Phi} \Phi)^{2}\right],
$$


with the temperature-dependent pre-factors $a(T)=a_{0}+a_{1}\left(\frac{T_{0}}{T}\right)+a_{2}\left(\frac{T_{0}}{T}\right)^{2}$ and $b(T)=b_{3}\left(\frac{T_{0}}{T}\right)^{3}$. In both versions the parameter $T_{0}=270 \mathrm{MeV}$ corresponds to the transition temperature in the pure YM theory. The third version, proposed by Fukushima, has only two parameters $a$ and $b$ and is inspired from a strong-coupling analysis

$$
\frac{\mathscr{U}_{\text {Fuku }}}{T^{4}}=-\frac{b}{T^{3}}\left[54 e^{-a / T} \Phi \bar{\Phi}+\ln \left(1-6 \Phi \bar{\Phi}-3(\Phi \bar{\Phi})^{2}+4\left(\Phi^{3}+\bar{\Phi}^{3}\right)\right)\right] .
$$

The parameter $a$ determines the deconfinement transition in pure gauge theory, while $b$ controls the mixing of the chiral and the deconfinement transition. Here, they are not fitted to the lattice data but also reproduce a first-order phase transition at $T_{0} \sim 270 \mathrm{MeV}$ in the pure YM theory. Details concerning the PQM model can be found in the forthcoming work [10].

When dynamical quarks are present in the system the running coupling of QCD is modified by fermionic contributions. The size of this effect can be estimated within perturbation theory, see e.g. [11]. As shown in Ref. [12] this leads to an $N_{f}$-modification of the expansion coefficients in the polynomial Polyakov-loop potential and can further be mapped onto an $N_{f}$-dependent $T_{0}$. In Tab. 1 one sees that the critical temperature $T_{0}$ decreases for increasing $N_{f}$.

\begin{tabular}{|c||c|c|c|c|c|}
\hline$N_{f}$ & 0 & 1 & 2 & $2+1$ & 3 \\
\hline$T_{0}[\mathrm{MeV}]$ & 270 & 240 & 208 & 187 & 178 \\
\hline
\end{tabular}

Table 1: The critical temperature $T_{0}$ for $N_{f}$ massless flavors according to [12]. The value for $2+1$ flavors has been estimated by using HTL/HDL theory for a massive strange quark with $m_{s}=150 \mathrm{MeV}$.

\subsection{Thermodynamic potential}

The thermodynamic potential of the PQM model is evaluated in mean-field approximation similar to [12, 13]. It splits into three contributions: the mesonic, $U\left(\sigma_{x}, \sigma_{y}\right)$, the quark/antiquark, $\Omega_{\bar{q} q}$, and the Polyakov loop contributions

$$
\Omega=U\left(\sigma_{x}, \sigma_{y}\right)+\Omega_{\bar{q} q}\left(\sigma_{x}, \sigma_{y}, \Phi, \bar{\Phi}\right)+\mathscr{U}(\Phi, \bar{\Phi}) .
$$

In general, the grand potential is a function of the temperature and three quark chemical potentials, one for each flavor. Here, we consider the isospin-symmetric case with two degenerated light quark masses. As a consequence, only two independent quark chemical potentials, the light $\mu_{q}$ and the strange $\mu_{s}$, emerge. Furthermore, only two order parameters in the meson sector, the non-strange $\sigma_{x}$ and strange $\sigma_{y}$ condensate are present. Note, the Polyakov loop variables are coupled to the fermionic part that also depends on the mesonic condensates via the quark masses. Finally, the temperature and quark chemical potential dependence of all four order parameters for the chiral and deconfinement transition are determined as solutions of the corresponding coupled equations of motion, i.e.,

$$
\frac{\partial \Omega}{\partial \sigma_{x}}=\frac{\partial \Omega}{\partial \sigma_{y}}=\frac{\partial \Omega}{\partial \Phi}=\left.\frac{\partial \Omega}{\partial \bar{\Phi}}\right|_{\min }=0
$$

where min $=\left(\sigma_{x}=\left\langle\sigma_{x}\right\rangle, \sigma_{y}=\left\langle\sigma_{y}\right\rangle, \Phi=\langle\Phi\rangle, \bar{\Phi}=\langle\bar{\Phi}\rangle\right)$ labels the global minimum of the grand potential.

In the following we have chosen a parameter set where both transitions coincide at $\mu=0$ as suggested by lattice data which we use for comparison [4]. This is the case for $m_{\sigma}=600 \mathrm{MeV}$ and $T_{0}=270 \mathrm{MeV}$ where all three Polyakov-loop potentials yield coinciding transitions. 


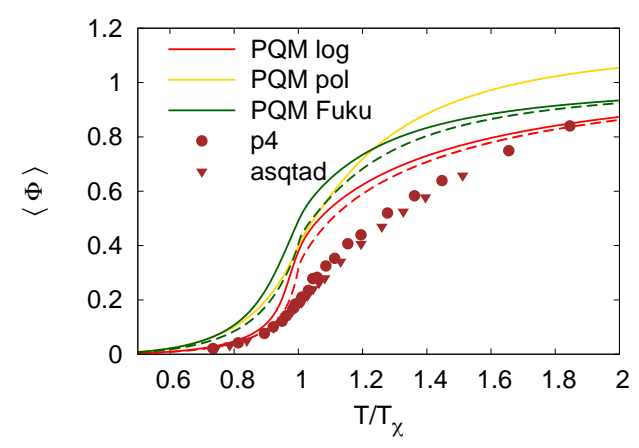

(a) Polyakov loop expectation value $\langle\Phi\rangle$

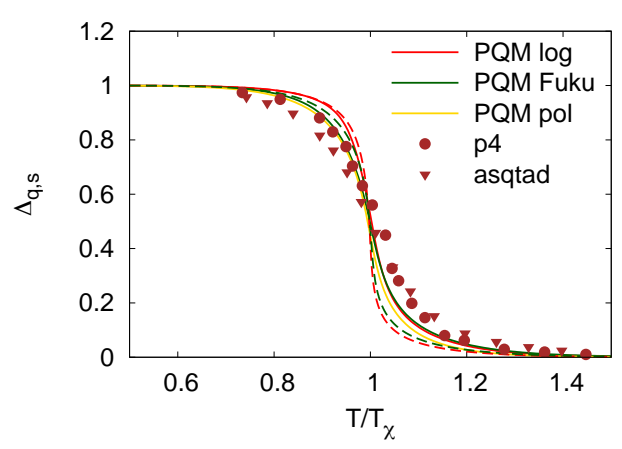

(b) Subtracted condensate $\Delta_{q, s}$

Figure 1: The Polyakov loop expectation value $\langle\Phi\rangle$ (left panel) and the subtracted condensate $\Delta_{q, s}$ (right panel) as a function of temperature for different Polyakov-loop potentials in comparison to lattice data for $N_{\tau}=8$ (symbols) [4]. Solid lines correspond to larger pion and kaon masses as used in the lattice simulations, dashed lines to physical masses.

\section{QCD thermodynamics}

In Fig. 1(a) the Polyakov loop expectation value $\langle\Phi\rangle$ is shown as a function of temperature for three different Polyakov loop potentials. For a proper comparison of the model results with the HotQCD [4] and RBC-Bielefeld lattice data [3], we also adjust the pion and kaon masses accordingly to $m_{\pi}=220 \mathrm{MeV}$ and $m_{K}=503 \mathrm{MeV}$. Heavier meson masses yield also slightly heavier constituent quark masses. The increased meson masses shift the transitions also to higher temperatures. The strongest shift is seen in the non-strange chiral transition since the quark masses are largest in this sector and both transitions, the non-strange and deconfinement one, still coincide. The strange sector is almost unaffected. Solid lines in Fig. 1(a) correspond to heavier meson masses, used in lattice simulations and dashed lines to the physical masses. The values are slightly higher for the Fukushima potential than for the logarithmic potential which exhibits a sharper crossover. The influence of the higher meson masses is mild. The logarithmic potential comes closet to the lattice data in the broken phase, while the difference between the model results and the lattice is considerable in the restored phase for all potentials.

For $\mu=0$ the Polyakov loop expectation value $\langle\Phi\rangle$ is available in model as well as in lattice calculations. This is in contrast to the chiral condensate $\langle\bar{q} q\rangle$. A direct comparison of $\langle\bar{q} q\rangle$ or $\langle\bar{s}\rangle\rangle$ with lattice simulations is difficult since unknown renormalization/normalization factors are involved. Therefore, a better quantity to compare with lattice simulations is the ratio

$$
\Delta_{q, s}=\frac{\langle\bar{q} q\rangle(T)-\left(\hat{m}_{q} / \hat{m}_{s}\right)\langle\bar{s} s\rangle(T)}{\langle\bar{q} q\rangle(0)-\left(\hat{m}_{q} / \hat{m}_{s}\right)\langle\bar{s} s\rangle(0)}
$$

which involves the non-strange $\langle\bar{q} q\rangle$ and strange $\langle\bar{s} s\rangle$ condensates, correspondingly $\left\langle\sigma_{x}\right\rangle$ and $\left\langle\sigma_{y}\right\rangle$ in our model calculation. Since the bare quark masses $\hat{m}_{q}, \hat{m}_{s}$ are not directly available, the ratios of the explicit symmetry breaking parameters of our model are used instead, cf. [15].

The results for $\Delta_{q, s}$ are shown in Fig. 1(b). The model calculations are in better agreement than for the Polyakov loop expectation value. However, also here, the lattice data exhibit a smoother transition than the model results. The logarithmic potential generates the sharpest transition while 


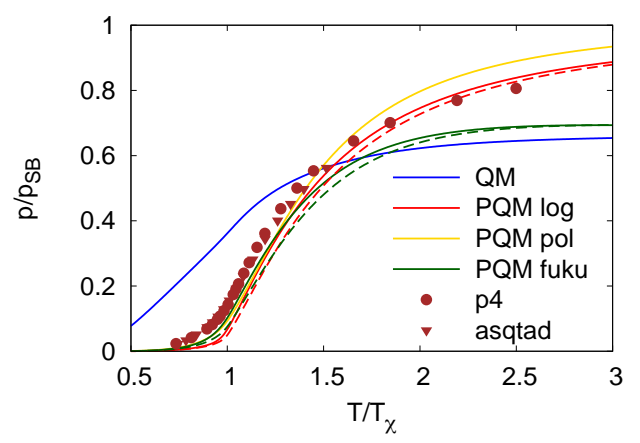

Figure 2: The normalized pressure as a function of temperature. The model calculations (PQM model with various Polyakov loop potentials and the QM model [14]) are compared to lattice data similar as in Fig. 1.

the Fukushima and polynomial potential are closer to the lattice results. The influence of the larger meson masses is stronger in the broken phase where the transition becomes smoother for larger meson masses.

The pressure $p(T, \mu)=-\Omega(T, \mu)$ is directly obtained from the thermodynamic potential with the normalization $p(0,0)=0$. In Fig. 2 the $(\mathrm{P}) \mathrm{QM}$ model pressures, normalized to the SB value of the PQM model, are compared to lattice data. As expected, the QM model [14] fails in describing the lattice data for all temperatures, while the PQM model results are in reasonable agreement with the data. The best agreement with the lattice data is achieved with Fukushima's potential but fails for temperatures above $1.5 T_{\chi}$. Physical meson (quark) masses (dashed lines) push the pressure to lower values, in particular around the transition.

\subsection{Taylor expansion and a novel algorithmic differentiation method}

As already mentioned one approach to overcome the sign problem in finite density QCD lattice simulations is based on an extrapolation from zero chemical potentials by a Taylor expansion. For the pressure the expansion in powers of $(\mu / T)$ at $\mu=0$ reads

$$
\frac{p(\mu / T)}{T^{4}}=\sum_{n=0}^{\infty} c_{n}(T)\left(\frac{\mu}{T}\right)^{n} \quad \text { with } \quad c_{n}(T)=\left.\frac{1}{n !} \frac{\partial^{n}\left(p(T, \mu) / T^{4}\right)}{\partial(\mu / T)^{n}}\right|_{\mu=0} .
$$

The coefficients $c_{n}(T)$ are accessible in QCD lattice simulations. Because of the CP-symmetry of the QCD partition function, $Z(\mu)=Z(-\mu)$, only even coefficients contribute. These have been calculated by different lattice groups for $N_{f}=2$ [16, 17] and $N_{f}=2+1$ quark flavors [18], currently up to order $n \leq 8$. For the realistic $2+1$ flavor scenario $N_{\tau}=4,6$ lattices have been used and higher coefficients still have significant errors [18].

In principle, these coefficients can be obtained without any limitations in model calculations. Doing this analytically is a tedious and difficult process. Furthermore, standard numerical techniques like divided differences fail since the errors increase rapidly. In order to proceed we have developed a novel numerical technique based on algorithmic differentiation (AD). This technique allows the evaluation of higher derivatives to extremely high precision. In fact, it is essentially limited only by machine precision. Details of this method can be found in Ref. [7]. 


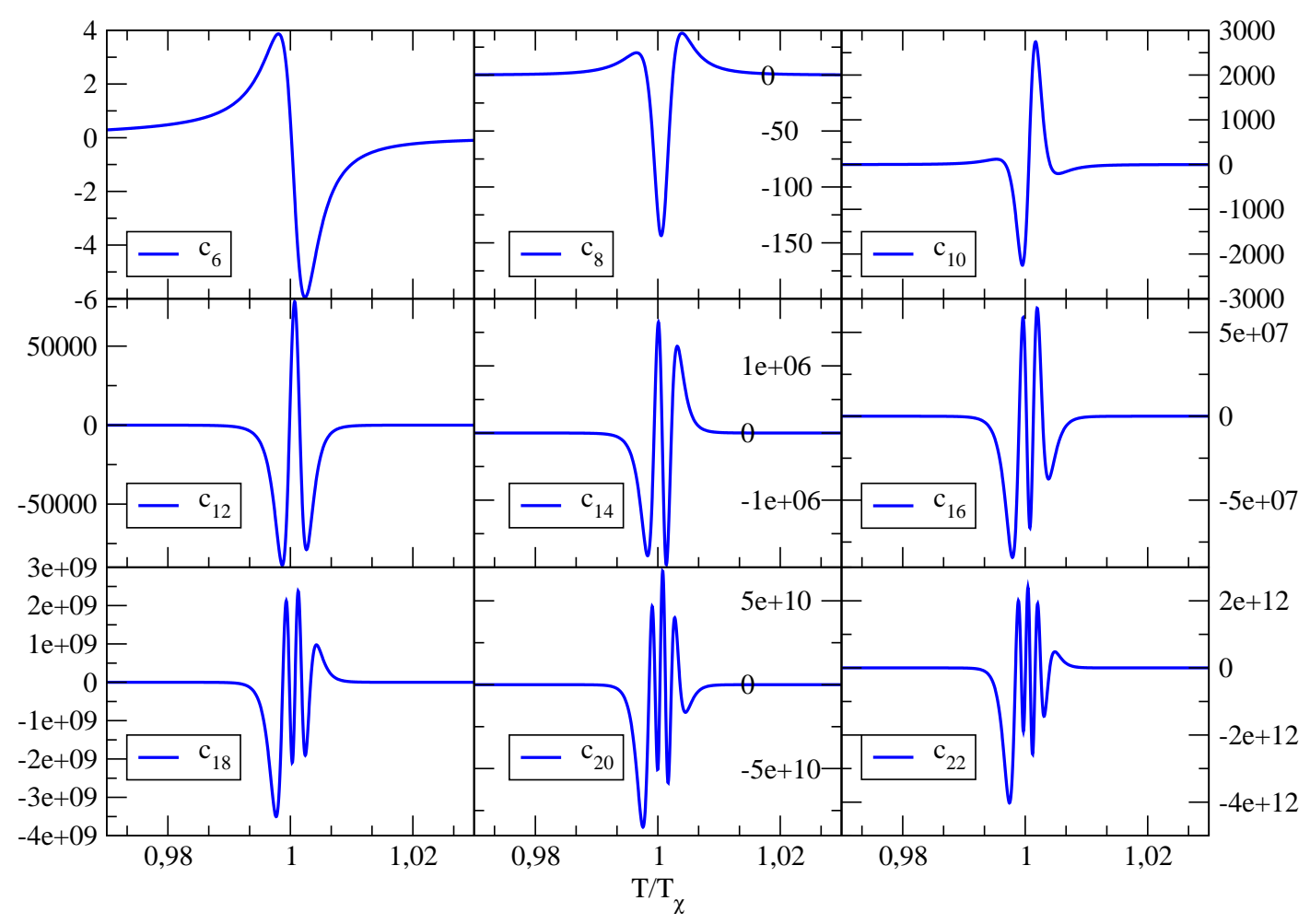

Figure 3: The Taylor coefficients $c_{6}$ to $c_{22}$ in the PQM model with the logarithmic Polyakov-loop potential.

In Fig. 3 we show the resulting coefficients $c_{6}$ to $c_{22}$ for the $2+1$ flavor PQM model with the logarithmic Polyakov loop potential as a function of temperature. With increasing order $n$, the coefficients $c_{n}$ start to oscillate around the transition temperature $T_{\chi}$. Inside a narrow temperature window around $T_{\chi}$, i.e., in the interval $0.95 T_{\chi} \gtrsim T \gtrsim 1.05 T_{\chi}$ the oscillation amplitude of the coefficients increases while they tend to zero far away of $T_{\chi}$. This means that higher order coefficients become more and more important in the expansion even for $\mu / T<1$ and the convergence of the series becomes questionable. Nevertheless, the Taylor expansion might still be useful to gain some information on the existence and location of a possible critical endpoint (CEP) in the QCD phase diagram. For some recent estimates by lattice groups using only a few coefficients see [16, 17]. However, the validity of these results and the convergence of the series remain questionable.

An often considered quantity to extract some information on the CEP is the quark number susceptibility $\chi_{q}=\left(\partial^{2} \Omega\right) /\left(\partial \mu^{2}\right)$. Since $\chi_{q}$ diverges exactly at the CEP it is a suitable quantity to locate this point at least in model calculations. Its expansion can be expressed with the same Taylor coefficients $c_{n}$ via

$$
\frac{\chi_{q}(\mu / T)}{T^{2}}=\sum_{n} n(n-1) c_{n}(T)\left(\frac{\mu}{T}\right)^{n-2} .
$$

In Fig. 4 we compare $\chi_{q}$ obtained with the Taylor expansion of different orders with the evaluation of the model. For the chosen parameters the CEP is located at $\left(T_{c}, \mu_{c}\right) \sim(185,167) \mathrm{MeV}$, i.e., at $\left(\mu_{c} / T_{c}\right) \sim 0.9$ (middle panel). The other two panels show $\chi_{q}$ for the ratios $(\mu / T)=0.8$ and 1.0, 


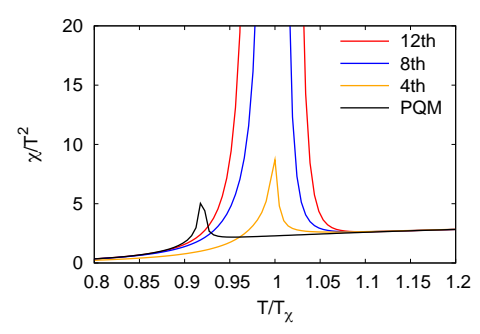

(a) $n=4,8,12,\left(\frac{\mu}{T}\right)=0.8$

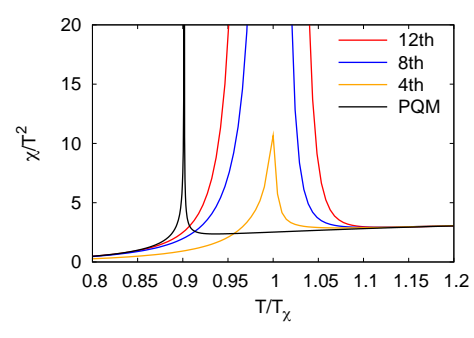

(b) $n=4,8,12,\left(\frac{\mu}{T}\right)=\left(\frac{\mu}{T}\right)_{c}$

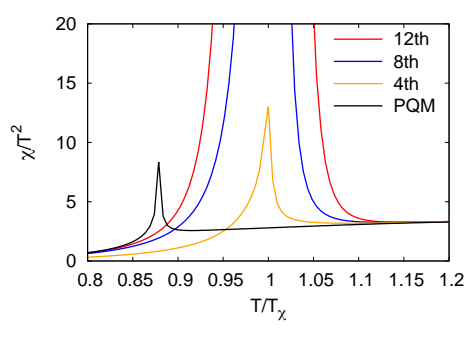

(c) $n=4,8,12,\left(\frac{\mu}{T}\right)=1.0$

Figure 4: The quark-number susceptibility $\chi_{q} / T^{2}$ for different ratios of $\mu / T$ and different orders of the Taylor expansion. The black line, labeled with 'PQM', is the model calculation.

respectively. As expected, $\chi_{q}$ diverges at $T_{c} \sim 0.9 T_{\chi}$ and is finite for larger or smaller ratios $(\mu / T)$ near the critical one (lines labeled with 'PQM'). Even the $12^{\text {th }}$ order expansion neither reproduces this structure nor shows any significant remnant of the divergence at the CEP. In the chirally broken phase, i.e., on the left side of the peak, the agreement becomes better with increasing orders of the Taylor expansion. The peak structure which is visible in the Taylor expansion around $T \sim T_{\chi}$ is a pure truncation artifact and a signal for its breakdown near the transition. On the other hand, in the chirally restored phase at high temperatures, the expansion again reproduces the model result, even for $\mu / T=1$. This is in agreement with the high-temperature limit of the coefficients $c_{n}$ since only the coefficients with $n \leq 4$ have a finite Stefan-Boltzmann value.

For a deeper understanding of the breakdown of the Taylor expansion it is instructive to consider its convergence radius. It can be obtained from the definition

$$
r=\lim _{n \rightarrow \infty} r_{2 n}=\lim _{n \rightarrow \infty}\left|\frac{c_{2 n}}{c_{2 n+2}}\right|^{1 / 2} .
$$

It is not known how well the radius $r$ is estimated by $r_{n}$ for a finite value $n$.

In Fig. 5 we show the convergence radius for different orders $n$ in the phase diagram together with the chiral and deconfinement phase boundaries. For temperatures close to $T_{\chi}$ the oscillations in the coefficients cause oscillations in $r_{n}$. For smaller temperature, i.e., approaching the CEP from above, the CEP lies inside the convergence region for lower $n$ values. For larger $n$ values $r_{n}\left(T_{c}\right)$ approaches the CEP and points to $\mu_{c}$ once $T_{c}$ is known. In the first order phase transition region, i.e., for $T<T_{c}$ the interpretation of the convergence radius $r_{n}$ becomes misleading. At a first order transition a new global minimum appears in the grand potential and the order parameter jumps to the new minimum. But the Taylor expansion is still performed around the $\mu=0$ minimum and cannot capture the jump. This means that its convergence radius is only valid for this $\mu=0$ minimum. Therefore the results obtained with the expansion are only valid up to the first order phase boundary.

Once $T_{c}$ is known, the convergence radius might provide an estimate for the critical $\mu_{c}$ and to locate the CEP. In [17] the critical temperature was estimated by using the sign of the Taylor coefficients. It contains information on the location of the CEP in the complex chemical potential plane [19]. For temperatures below the critical one all coefficients should be positive and the first root in the coefficients for $T<T_{\chi}$ should determine $T_{c}$ in the limit $n \rightarrow \infty$. Since the location of 


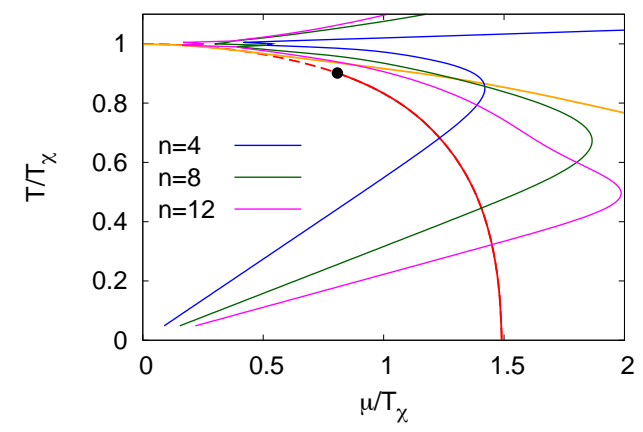

(a) $n=4,8,12$

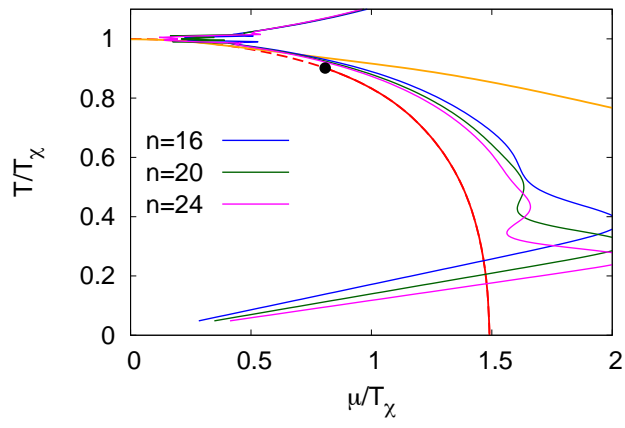

(b) $n=16,20,24$

Figure 5: The convergence radii $r_{n}$ for different orders $n$ of the expansion in the PQM model with the logarithmic Polyakov loop potential $\left(T_{0}=270 \mathrm{MeV}\right)$. Also shown are the phase boundaries for the chiral transition (red line; dashed: crossover, solid: first order) and the deconfinement crossover transition (yellow line). The black dot indicates the CEP.

the CEP is precisely known in the model calculation we can verify this idea. From the coefficients, shown in Fig. 3, we observe that the first roots of the coefficients, starting at $T \sim T_{\chi}$ for $n=6$, stay far away from the critical temperature $T_{c} \sim 0.9 T_{\chi}$. From this one might conclude, that the Taylor expansion yields a too large value for the critical temperature. A more detailed study in this direction is underway [20].

\section{Summary}

In this talk we have presented recent work, which extends a two flavor Polyakov-quark-meson model to $N_{f}=(2+1)$ quark flavors. The bulk thermodynamic of this model in mean-field approximation is compared to recent $(2+1)$-flavour QCD lattice simulations. The larger quark masses on the lattice are also considered in the model calculations. For the Polyakov-loop potential we examine three different ansätze which all reproduce a first-order phase transition in the pure gauge sector at the critical temperature $T_{c}=270 \mathrm{MeV}$. For the used parameter sets of the Polyakov-loop potentials the chiral and the deconfinement transition coincide at vanishing chemical potential. With the Polyakov-loop a very good agreement of the QCD equation of state in particular in the transition region up to temperature of $T \sim 1.5 T_{\chi}$ is achieved in contrast to a pure three flavor quark-meson model.

Furthermore, we analyze the finite chemical potential expansion of the quark-number susceptibility in a Taylor series around vanishing chemical potential. By means of a novel algorithmic differentiation technique, we calculate the Taylor coefficients up to $24^{\text {th }}$ order in the PQM model for the first time. The knowledge of these higher Taylor coefficients allows the systematic study of convergence properties of the series. The peak in the quark-number susceptibility $\chi_{q}$, seen in the Taylor expansion, is a pure artifact and related to the breakdown of the expansion. In particular, the divergence of $\chi_{q}$ at the critical endpoint in the phase diagram cannot be captured by the Taylor series. Nevertheless, away from the phase transition the Taylor expansion converges rapidly and coincides with the model evaluation even for $\mu / T=1$. In the first-order region discontinuities in 
the order parameter emerge which cannot be captured by the Taylor series either. However, our analysis of the convergence radius yields that the expansion might work even for $\mu / T>1$ if one stays in the broken phase. But in lattice simulations this transition boundary is not known. Thus, in order to make statements on the possible location of a critical endpoint in the phase diagram with the Taylor expansion technique our studies suggest that higher order coefficients with $n>8$, more as currently available on the lattice, are definitely required.

Acknowledgments The work of MW was supported by the Alliance Program of the Helmholtz Association (HA216/ EMMI) and BMBF grants 06DA123 and 06DA9047I. JW was supported in part by the Helmholtz International Center for FAIR.

\section{References}

[1] M. A. Stephanov, PoS LAT2006, 024 (2006).

[2] L. McLerran and R. D. Pisarski, Nucl. Phys. A796, 83 (2007).

[3] M. Cheng et al., Phys. Rev. D74, 054507 (2006); M. Cheng et al., Phys. Rev. D77, 014511 (2008).

[4] A. Bazavov et al., Phys. Rev. D80, 014504 (2009).

[5] Y. Aoki et al., Phys. Lett. B643, 46 (2006); Y. Aoki et al., JHEP 06, 088 (2009).

[6] C. Schmidt, PoS LAT2006, 021 (2006); O. Philipsen, Eur. Phys. J. Spec. Top. 152, 29 (2007).

[7] M. Wagner, A. Walther, and B.-J. Schaefer, subm. to Comput. Phys. Commun. (2009).

[8] B.-J. Schaefer and M. Wagner, Progress in Particle and Nuclear Physics 62, 381 (2009).

[9] C. Ratti, M. A. Thaler, and W. Weise, Phys. Rev. D73, 014019 (2006); S. Roessner, C. Ratti, and W. Weise, Phys. Rev. D75, 034007 (2007); K. Fukushima, Phys. Rev. D77, 114028 (2008).

[10] B.-J. Schaefer, M. Wagner, and J. Wambach, arXiv:0910.5628 [hep-ph] .

[11] J. Braun and H. Gies, Phys. Lett. B645, 53 (2005); J. Braun and H. Gies, JHEP 06, 024 (2006).

[12] B.-J. Schaefer, J. M. Pawlowski, and J. Wambach, Phys. Rev. D76, 074023 (2007).

[13] O. Scavenius, A. Mocsy, I. N. Mishustin, and D. H. Rischke, Phys. Rev. C64, 045202 (2001).

[14] B.-J. Schaefer and M. Wagner, Phys. Rev. D79, 014018 (2009).

[15] H. Meyer-Ortmanns and B.-J. Schaefer, Phys. Rev. D53, 6586 (1996).

[16] C. R. Allton et al., Phys. Rev. D71, 054508 (2005).

[17] R. V. Gavai and S. Gupta, Phys. Rev. D71, 114014 (2005); R. V. Gavai and S. Gupta, Phys. Rev. D78, 114503 (2008).

[18] C. Miao and C. Schmidt (RBC-Bielefeld), PoS LAT2008, 172 (2008).

[19] M. A. Stephanov, Phys. Rev. D73, 094508 (2006).

[20] F. Karsch, B.-J. Schaefer, M. Wagner, and J. Wambach, in preparation (2009). 